\title{
Development and characterization of sugar palm nanocrystalline cellulose reinforced sugar palm starch bionanocomposites
}

\begin{abstract}
Sugar palm fibre (SPF) was treated with $\mathrm{NaClO}_{2}$, bleached with $\mathrm{NaOH}$ and subsequently hydrolyzed with acid to obtain sugar palm nanocrystalline cellulose (SPNCCs). Bionanocomposites in the form of films were prepared by mixing sugar palm starch (SPS) and sorbitol/glycerol with different nanofiller SPNCCs compositions (0-1.0 wt\%) using solution casting method. The resulting fibres and nanocomposites were characterized in terms of morphology (FESEM and TEM), footprint, crystallinity (XRD), light transmittance, biodegradability, physical, water barrier, thermal (TGA, DSC and DMA) and mechanical properties. The length (L), diameter (D) and L/D values of the SPNCCs were $130 \pm 30.23$, $8.5 \pm 1.82 \mathrm{~nm}$, and 15.3, respectively. The SPS/SPNCCs nanocomposite films exhibited higher crystallinity, tensile strength, Young's modulus, thermal and water-resistance compared to the neat SPS film. The results showed that the tensile strength and moduli of the bionanocomposites increased after being reinforced with SPNCCs and the optimum nanofiller content was $0.5 \%$.
\end{abstract}

Keyword: Sugar palm; Starch; Nanocrystalline cellulose; Biodegradable films; Nanocomposites; Mechanical properties 